\title{
Condition Assessment for Wind Turbines with Doubly Fed Induction Generators Based on SCADA Data
}

\author{
Peng Sun*, Jian $\mathrm{Li}^{\dagger}$, Caisheng Wang** and Yonglong Yan***
}

\begin{abstract}
This paper presents an effective approach for wind turbine (WT) condition assessment based on the data collected from wind farm supervisory control and data acquisition (SCADA) system. Three types of assessment indices are determined based on the monitoring parameters obtained from the SCADA system. Neural Networks (NNs) are used to establish prediction models for the assessment indices that are dependent on environmental conditions such as ambient temperature and wind speed. An abnormal level index (ALI) is defined to quantify the abnormal level of the proposed indices. Prediction errors of the prediction models follow a normal distribution. Thus, the ALIs can be calculated based on the probability density function of normal distribution. For other assessment indices, the ALIs are calculated by the nonparametric estimation based cumulative probability density function. A Back-Propagation NN (BPNN) algorithm is used for the overall WT condition assessment. The inputs to the BPNN are the ALIs of the proposed indices. The network structure and the number of nodes in the hidden layer are carefully chosen when the BPNN model is being trained. The condition assessment method has been used for real 1.5 MW WTs with doubly fed induction generators. Results show that the proposed assessment method could effectively predict the change of operating conditions prior to fault occurrences and provide early alarming of the developing faults of WTs.
\end{abstract}

Keywords: Condition assessment, Prediction model, Neural network, SCADA data, Wind turbine

\section{Introduction}

Wind energy is considered one of the most promising alternatives to traditional electric power systems $[1,2]$. Rapid expansion of large-scale wind farms has drawn attention to operational and maintenance (O\&M) issues [3-5]. The O\&M costs of wind turbines (WTs) account for approximately $25 \%$ to $30 \%$ of the overall energy generation cost [6]. Various condition monitoring and fault diagnosis approaches of WTs, such as vibration analysis [7], acoustic analysis [8], lubrication analysis [9], and strain measurement [10], have been proposed to reduce unscheduled downtime as well as O\&M costs. However, most of these approaches have not been widely adopted in wind farms because of the imitations with data storage capacities and high cost of installing additional equipment, or sensors, for condition monitoring systems of WTs [11]. As one of the most important parts of the WT condition monitoring systems, the supervisory control and data acquisition (SCADA) system of a wind farm can provide a large amount of measurements such as the temperatures

$\dagger$ Corresponding Author: State Key Laboratory of Power Transmission Equipment and System Security and New Technology, Chongqing University, China. (lijian@cqu.edu.cn)

* State Grid Henan Electrical Power Research Institute, Zhengzhou 450000, China.

** Department of Electrical and Computer Engineering, Wayne State University, Detroit, MI 48202, USA.

*** State Grid Wulumuqi Electric Supply Company, Wulumuqi 830011, China.

Received: April 19, 2016; Accepted: October 28, 2016 (e.g., bearing temperature, oil temperature), wind parameters (e.g., wind speed, wind direction), and energy conversion parameters (e.g., output power, pitch angle, rotor speed), which are widely used by wind farm operators to monitor the health condition of WTs. Hence, assessment of WT conditions based on the SCADA data is a cost-effective approach to improving the reliability of WTs and reducing the O\&M costs of wind farms $[12,13]$.

The wind farm SCADA data has gained considerable interest in condition monitoring and fault diagnosis of WTs [14]. The WT power curve, which could provide the relationship between power output and wind speed, is one of the most common tools in anomaly detection and performance analysis of WTs [15]. Different SCADA data mining approaches, such as neural networks (NNs) [16, 17], adaptive neuron-fuzzy inference systems (ANFIS) [17], $k$-Nearest Neighbors $(k-\mathrm{NN})[17,18]$, bagging tree [18], and the generalized mapping regressor [19], PC2-Dev [20] were used for modeling the WT power curve and the anomaly detection capabilities of the models were analyzed. It was shown that the abnormal conditions of the WTs can be detected by using the residuals between the predicted power and observed power. In [21], a methodology for system-level fault diagnosis of WTs was presented by using the SCADA based power curve and a NN classifier. A comprehensive review of the existing WT power curve monitoring techniques can be found in [22].

Modeling the normal behavior of SCADA monitoring parameters is another effective method for WT condition 
monitoring [23]. By using advanced SCADA data mining methods, various models predicting normal behavior have been developed to detect common anomalies in most important WT components. These models were established by employing data-driven approaches that involve NNs [24-26], support vector machine (SVM) [27], ANFIS [28], deep neural network (DNN) [29], and nonlinear state estimate technique [30]. In [31], different WT performance curves, such as the power curve, rotor curve, and blade pitch curve were modeled for monitoring performance of WTs. A nonlinear data-based modeling approach was proposed in [32] to detect anomalies in WT generator winding and gearbox bearing. In [33], a NN based normal behavior model of generator bearing temperature was developed to analyze bearing faults in WTs. A comparative analysis of two NN-based models and a regression-based model was presented in [34] for detecting anomalies in gearbox bearing temperature and generator stator temperature.

Though the wind farm SCADA data have attracted great research interest in anomaly detection and condition monitoring for WTs, there is still a lack of the assessment method for monitoring the overall condition of WTs. The power curve monitoring techniques are unable to utilize the comprehensive operational information hidden within the SCADA system for condition assessment of WTs. Though normal behavior modeling is a viable approach to performance monitoring of WTs, the technique is mainly applied to monitor the conditions of single signal or individual WT components such as blades, rotors, generators, and gearboxes, which are not appropriate to be used for assessing the overall condition of WTs. These challenges lead to the discovery of a new approach to evaluate the operation conditions of WTs based on comprehensive SCADA monitoring parameters. Moreover, since the operational point of a WT change quite significantly with wind speed [11], the traditional fixed thresholds based condition assessment method $[35,36]$ is not appropriate for WTs. Data preprocessing methods were proposed in $[23,37]$ to mitigate the impacts of wind speed upon the real WT condition parameters. However, other factors such as the ambient temperature, yaw control and pitch angle control can also affect the performance of the WTs $[18,38]$. Therefore, the environmental and operational conditions should be comprehensively considered to quantify the condition assessment indices effectively.

Considering the aforementioned problems, this paper presents a novel approach for condition assessment of WTs. Based on the monitoring parameters of a wind farm SCADA system, the indices for WT condition assessment were determined and classified into three types. The development of prediction models of rotor speed, output power, and component temperature is given to extract useful information from the assessment indices that are dependent on environmental conditions such as ambient temperature and wind speed. A new index called abnormal level index (ALI) is proposed to quantify the abnormal level of the proposed indices. A BPNN algorithm is used for the WT condition assessment with the ALI as inputs. Finally, two cases are investigated to validate the proposed method by using the monitoring data of $1.5 \mathrm{MW}$ WTs.

The remainder of the paper is organized as follows: In Section 2, the indices based on the SCADA data have been determined for WT condition assessment. The prediction models of the assessment indices are developed and their performance is discussed in Section 3. In Section 4, different data preprocessing methods are used to calculate the ALIs of the indices. The BPNN model for WT condition assessment is introduced in Section 5, where the details in selecting the structure of the BPNN model and the NN training are also given. Based on the data of a real wind farm, the BPNN based condition assessment method is verified and discussed in Section 6, followed by the conclusion drawn in Section 7.

\section{Parameter Description and Classification}

Hundreds of parameters are provided by the wind farm SCADA systems. Fig. 1 shows an example of some WT condition parameters obtained from the wind farm SCADA system. All these parameters can be classified into component performance, environmental, and grid parameters. The operational condition of components is reflected by the component performance parameters, such as temperature, pressure, and position. Typical environmental parameters are wind speed, wind direction, and ambient temperature. Grid parameters include phase voltage, phase current, grid frequency, reactive power, and output power. Only the parameters that can reflect the health condition of a WT are considered in this paper. Defects and fatigue in electronic equipment or mechanical components can be reflected in temperature parameters $[33,39]$. For some subsystems of a WT, such as yaw and hydraulic system, other physical quantities should be utilized. Yaw angle error represents the angle between the wind and the nacelle position. Yaw

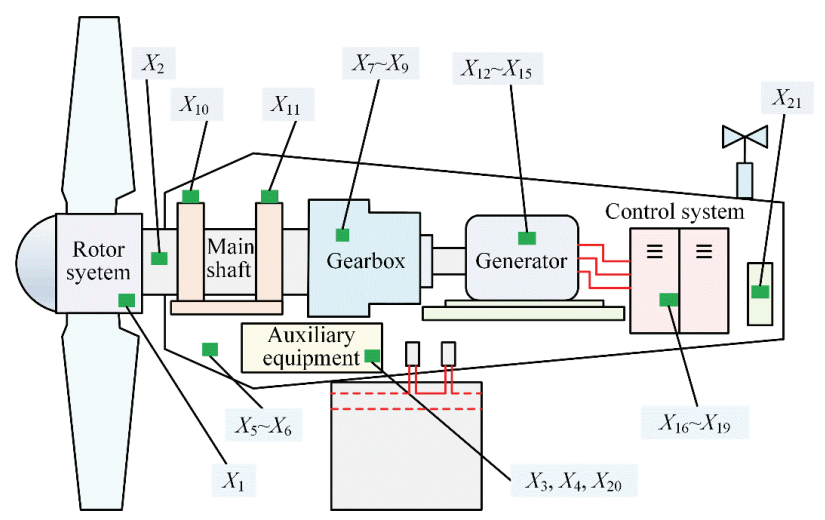

Fig. 1. Location of part of measurements by the SCADA system 
position is the rotation angle of the nacelle. The output power is also selected since it can reflect the overall performance of a WT.

Based on this, the SCADA based WT condition assessment indices are determined, as shown in Table 1. All the indices in Table 1 are grouped into three types. Type 1 indices include the temperature indices for WT components that are affected by both wind speed and ambient temperature. Temperature of the gearbox input shaft in the wind farm is taken as an example. As shown in Fig. 2, for the variable speed constant-frequency (VSCF) WT, when the wind speed is below its rated speed, a faster rotational speed due to a higher wind speed will evidently raise the temperature of the mechanical components. When the wind speed is over the rated wind speed, the WT will be kept at its rated output power by variable pitch control and the component

Table 1. WT condition assessment indices.

\begin{tabular}{|c|c|c|c|}
\hline Components & Assessment indices & Unit & Types \\
\hline \multirow{2}{*}{ Rotor } & Rotor position- $X_{1}$ & 0 & 3 \\
\hline & Rotor speed- $X_{2}$ & $\mathrm{rpm}$ & 2 \\
\hline \multirow{2}{*}{ Yaw } & Yaw position- $X_{3}$ & 0 & 3 \\
\hline & Yaw angle error- $X_{4}$ & $\circ$ & 3 \\
\hline \multirow{2}{*}{ Nacelle } & Nacelle vibration along $x$ direction- $X_{5}$ & $\mathrm{~m} / \mathrm{s}^{2}$ & 3 \\
\hline & Nacelle vibration along $y$ direction- $X_{6}$ & $\mathrm{~m} / \mathrm{s}^{2}$ & 3 \\
\hline \multirow{3}{*}{ Gearbox } & Temp. of gearbox input shaft- $X_{7}$ & ${ }^{\circ} \mathrm{C}$ & 1 \\
\hline & Temp. of gearbox output shaft- $X_{8}$ & ${ }^{\circ} \mathrm{C}$ & 1 \\
\hline & Temp. of gearbox oil- $X_{9}$ & ${ }^{\circ} \mathrm{C}$ & 1 \\
\hline \multirow{2}{*}{$\begin{array}{c}\text { Main shaft } \\
\text { bearing }\end{array}$} & Temp. of rotor-side main bearing- $X_{10}$ & ${ }^{\circ} \mathrm{C}$ & 1 \\
\hline & $\begin{array}{l}\text { Temp. of gearbox-side main bearing- } \\
\qquad X_{11}\end{array}$ & ${ }^{\circ} \mathrm{C}$ & 1 \\
\hline \multirow{4}{*}{ Generator } & Temp. of generator winding- $X_{12}$ & ${ }^{\circ} \mathrm{C}$ & 1 \\
\hline & Temp. of generator bearing $\boldsymbol{a}-X_{13}$ & ${ }^{\circ} \mathrm{C}$ & 1 \\
\hline & Temp. of generator bearing $\boldsymbol{b}-X_{14}$ & ${ }^{\circ} \mathrm{C}$ & 1 \\
\hline & Temp. of generator cooling air- $X_{15}$ & ${ }^{\circ} \mathrm{C}$ & 1 \\
\hline \multirow{4}{*}{$\begin{array}{l}\text { Control } \\
\text { system }\end{array}$} & Temp. of converter cooling water- $X_{16}$ & ${ }^{\circ} \mathrm{C}$ & 1 \\
\hline & Temp. of converter controller- $X_{17}$ & ${ }^{\circ} \mathrm{C}$ & 1 \\
\hline & Temp. of top cabinet- $X_{18}$ & ${ }^{\circ} \mathrm{C}$ & 1 \\
\hline & Temp. of base cabinet- $X_{19}$ & ${ }^{\circ} \mathrm{C}$ & 1 \\
\hline $\begin{array}{l}\text { Hydraulic } \\
\text { system }\end{array}$ & Main hydraulic pressure- $X_{20}$ & bar & 3 \\
\hline Grid factors & Output power- $X_{21}$ & $\mathrm{~kW}$ & 2 \\
\hline
\end{tabular}
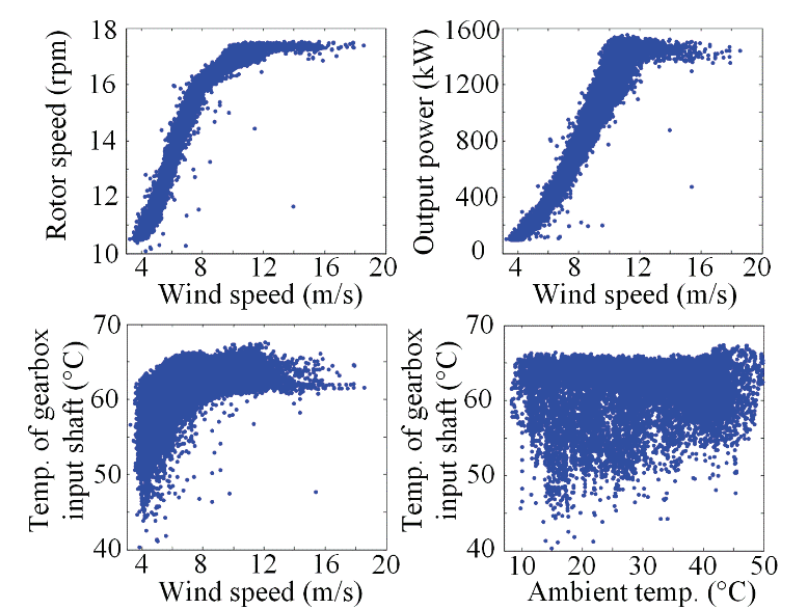

Fig. 2. Raw SCADA data obtained from a real 1.5 MW WT temperatures will be less affected by the wind speed. The heat dissipation of mechanical components of a WT is also affected by the ambient temperature. At a lower ambient temperature, the heat dissipation is faster and the component temperatures can vary in a larger range. Conversely, at a higher temperature, the component temperature tolerable variation range is smaller with slower heat dissipation. Type 2 indices consist of the WT's output power and rotor speed, which are closely related to the change of wind speed (Fig. 2). The indices in Type 3 do not have an obvious relationship with environmental conditions.

The SCADA data used in this paper were obtained from an onshore wind farm in Northern China. The wind farm has 34 1.5 MW WTs with doubly fed induction generators (DFIGs). The cut-in, the rated and the cut-out wind speeds of each WT are $3 \mathrm{~m} / \mathrm{s}, 12 \mathrm{~m} / \mathrm{s}$, and $25 \mathrm{~m} / \mathrm{s}$, respectively. The SCADA data have been collected since February 15, 2011 .

\section{Prediction model development}

Certain data processing steps are necessary to mitigate the impacts of dynamic operating points upon the WT condition parameters. Prediction models of the Type 1 and Type 2 indices are developed to decouple the indices from environmental and operational conditions. To ensure satisfactory estimation results, the following three factors should be carefully considered: 1) input parameters, 2) training algorithm, and 3) sample data. The input parameters to each model are determined based on domain knowledge. NNs are used to establish the prediction models for environmentally sensitive SCADA parameters (i.e. Type 1 and Type 2 indices). The sample data are collected when the WT is considered healthy.

\subsection{Input parameters}

The selection of input parameters is a premise in simplifying models and ensuring prediction accuracy. It requires a physical understanding of energy conversion of WTs with DFIGs. The input parameters of different types of parameter prediction models are shown in Table 2,

Table 2. Input parameters of the prediction models based on Type 1 and Type 2 assessment indices.

\begin{tabular}{c|c}
\hline Target condition parameters & Input parameters \\
\hline & Wind speed $(t) ;$ \\
Output power $(t)$ & yaw angle error $(t) ;$ \\
& pitch angle $(t)$ \\
\hline Rotor speed $(t)$ & Wind speed $(t) ;$ \\
& yaw angle error $(t) ;$ \\
pitch angle $(t)$
\end{tabular}


which differ for each type of monitoring parameter. Yaw control and pitch angle control can change the power captured by the blades and consequently affect the output power and the rotor speed of a WT [40]. The wind speed, yaw angle error, and the pitch angle are selected as the input parameters to improve the accuracy of the models of output power and rotor speed. For the VSCF WTs, the component temperatures depend on the wind speed, the ambient temperature, the WT's output power and the previous component temperatures. The temperature of the mechanical components will increase under a faster rotational speed caused by a higher wind speed. Consequently, the WT output power and the temperature of electrical components increase. The ambient temperature and previous temperature are considered due to the hysteresis of temperature variation.

\subsection{Training algorithm}

Back-propagation Neural Network (BPNN), a widely used NN for prediction, is applied in this study for prediction of Type 1 and Type 2 assessment indices. In consideration of the size of the training set and the training time, the $\mathrm{NN}$ is chosen to have only one hidden layer and the number of neurons in the hidden layer ranges from 2 to 10. The transfer function used in the hidden layer is tansigmoid while the output layer transfer function is logsigmoid based. The actual number of nodes (i.e. neurons) in the hidden layer is determined by trial-and-error to find the right number at which the $\mathrm{NN}$ has the best generalization performance.

\subsection{Data sampling}

There are no generalized rules for selecting training samples of the WT condition parameter prediction models. Two points have been carefully considered to select the sample data. Firstly, the training samples must be collected when the WT is under normal condition. Secondly, ambient temperature and wind speed vary with the seasons. Fig. 3 shows the boxplot of ambient temperature and wind speed of four seasons in a year. The data are collected from the SCADA dataset of a WT. The median, 25th and 75th
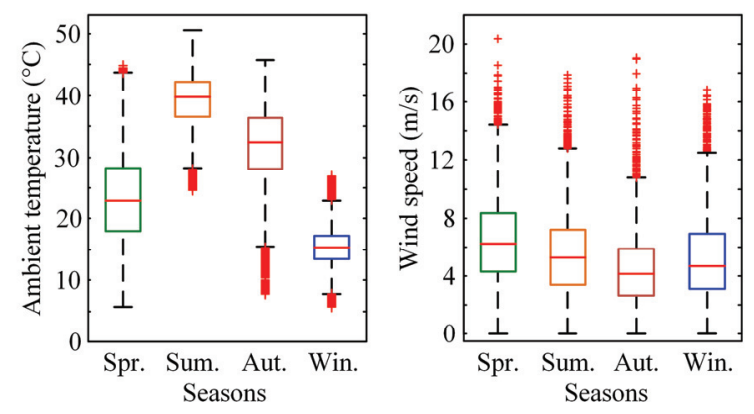

Fig. 3. Boxplot of ambient temperature and wind speed of four seasons percentiles are shown with boxes, while the maximum, minimum and outliers are shown with whiskers and crosses, respectively. It is clear that there are significant differences among the data distributions of the four seasons.

Table 3 shows the training and test data for the prediction models of the Type 1 and Type 2 assessment indices. The samples are collected from the SCADA data in 2011 and 2012. Data of each season are used to develop the prediction models. Scaling and missing data processing are performed before training the NNs. The 10 min average SCADA data are used for parameter modeling.

\subsection{Performance and prediction error analysis}

To analyze the performance of the prediction models, metrics such as mean absolute error (MAE), mean squared error (MSE), mean absolute percentage error (MAPE) and coefficient of determination $\left(\mathrm{R}^{2}\right)$ have been used, as shown in (1)-(3).

$$
\begin{gathered}
M A E=\frac{1}{n} \sum_{i=1}^{n}\left|\hat{y}_{i}-y_{i}\right| \\
M S E=\sqrt{\frac{1}{n} \sum_{i=1}^{n}\left(\hat{y}_{i}-y_{i}\right)^{2}} \\
M A P E=\frac{1}{n} \sum_{i=1}^{n}\left|\frac{\hat{y}_{i}-y_{i}}{y_{i}}\right| \times 100 \%
\end{gathered}
$$

Table 3. Data sample for the prediction models.

\begin{tabular}{cccc}
\hline \multirow{2}{*}{ Seasons } & \multicolumn{2}{c}{ Sample data } & \multirow{2}{*}{ Sample type } \\
\cline { 2 - 3 } & Start time & Stop time & \\
\hline \multirow{2}{*}{ Spring } & $2012-02-15$ & $2012-04-23$ & training \\
& $2012-04-24$ & $2012-04-30$ & test \\
\hline \multirow{2}{*}{ Summer } & $2011-05-01$ & $2011-07-24$ & training \\
& $2011-07-25$ & $2011-07-31$ & test \\
\hline \multirow{2}{*}{ Autumn } & $2011-08-01$ & $2011-10-24$ & training \\
& $2011-10-25$ & $2011-10-31$ & test \\
\hline \multirow{2}{*}{ Winter } & $2011-11-01$ & $2012-01-24$ & training \\
& $2012-01-25$ & $2012-01-31$ & test \\
\hline
\end{tabular}

Table 4. Prediction performance of Type 1 and Type 2 assessment indices.

\begin{tabular}{c|c|c|c}
\hline \multirow{2}{*}{ Assessment indices } & \multicolumn{3}{|c}{ Index } \\
\cline { 2 - 4 } & MSE & MAE & MAPE \\
\hline Temp. of gearbox input shaft & $0.45^{\circ} \mathrm{C}$ & $0.34^{\circ} \mathrm{C}$ & $0.58 \%$ \\
\hline Temp. of gearbox output shaft & $0.70^{\circ} \mathrm{C}$ & $0.51^{\circ} \mathrm{C}$ & $0.83 \%$ \\
\hline Temp. of gearbox oil & $0.83^{\circ} \mathrm{C}$ & $0.64^{\circ} \mathrm{C}$ & $1.09 \%$ \\
\hline Temp. of rotor-side main bearing & $0.28^{\circ} \mathrm{C}$ & $0.13^{\circ} \mathrm{C}$ & $0.45 \%$ \\
\hline Temp. of gearbox-side main bearing & $0.46^{\circ} \mathrm{C}$ & $0.15^{\circ} \mathrm{C}$ & $0.54 \%$ \\
\hline Temp. of generator winding & $1.81^{\circ} \mathrm{C}$ & $0.84^{\circ} \mathrm{C}$ & $1.67 \%$ \\
\hline Temp. of generator bearing $\boldsymbol{a}$ & $0.71^{\circ} \mathrm{C}$ & $0.29^{\circ} \mathrm{C}$ & $0.96 \%$ \\
\hline Temp. of generator bearing $\boldsymbol{b}$ & $0.68^{\circ} \mathrm{C}$ & $0.52^{\circ} \mathrm{C}$ & $0.88 \%$ \\
\hline Temp. of generator cooling air & $0.90^{\circ} \mathrm{C}$ & $0.59^{\circ} \mathrm{C}$ & $2.06 \%$ \\
\hline Temp. of converter cooling water & $1.05^{\circ} \mathrm{C}$ & $0.77^{\circ} \mathrm{C}$ & $1.24 \%$ \\
\hline Temp. of converter controller & $0.72^{\circ} \mathrm{C}$ & $0.51^{\circ} \mathrm{C}$ & $1.19 \%$ \\
\hline Temp. of top cabinet & $0.63^{\circ} \mathrm{C}$ & $0.79^{\circ} \mathrm{C}$ & $1.05 \%$ \\
\hline Temp. of base cabinet & $0.88^{\circ} \mathrm{C}$ & $0.67^{\circ} \mathrm{C}$ & $0.94 \%$ \\
\hline Output Power & $43.48 \mathrm{~kW}$ & $38.25 \mathrm{~kW}$ & $7.79 \%$ \\
\hline Rotor speed & $0.37 \mathrm{rpm}$ & $0.20 \mathrm{rpm}$ & $1.52 \%$ \\
\hline & & &
\end{tabular}


where $n$ is the number of test samples, $\hat{y}_{i}$ is the predicted value for a time period $i$, and $y_{i}$ is the measured value at the same time.

Tables 4 shows the prediction performance of Type 1 and Type 2 indices of a WT. Although the prediction accuracy of each component temperature is different, the maximum of MAE is lower than $1^{\circ} \mathrm{C}$.

The trained models are tested by using the test data in Table 3. The error residuals and prediction error distributions for the prediction models of output power, rotor speed, gearbox input shaft temperature and generator

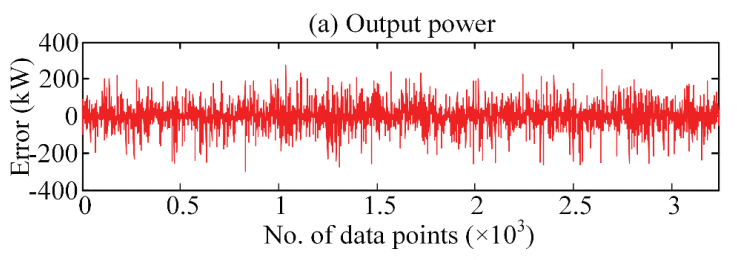

(b) Rotor speed

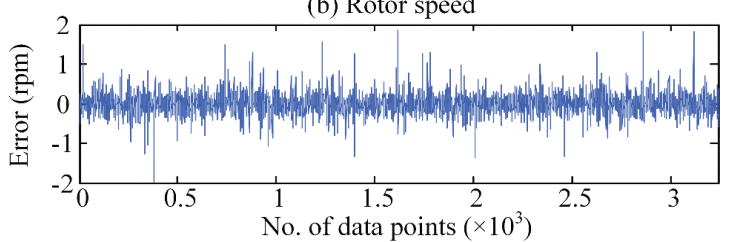

(c) Gearbox input shaft temperature

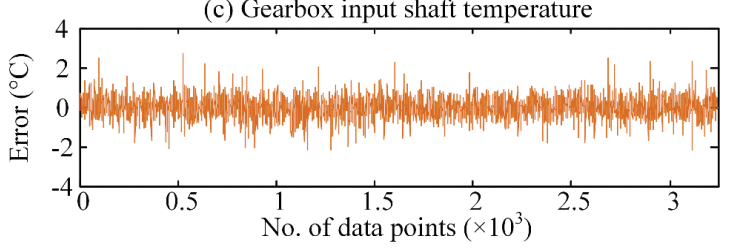

(d) Generator bearing temperature

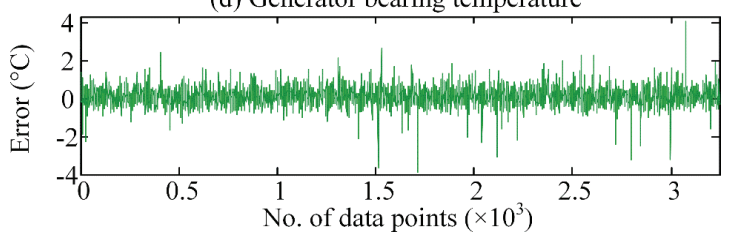

Fig. 4. Error residuals of the prediction models based on Type 1 and Type 2 indices
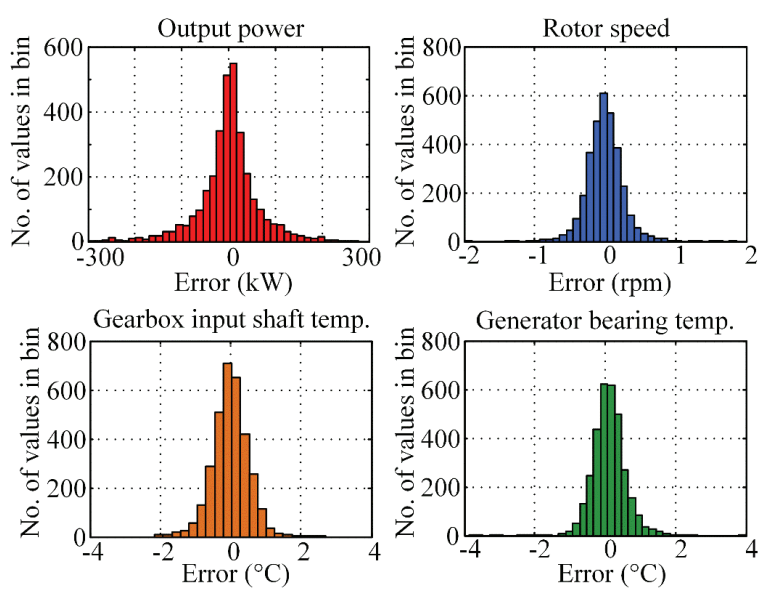

Fig. 5. Prediction error distributions of the prediction models based on Type 1 and Type 2 indices bearing temperature are given as an example, as shown in Figs. 4 and 5. It can be seen that the prediction errors coming from the successfully trained prediction models are normally distributed with a mean around zero. When the WT is healthy, the prediction errors are mostly distributed in high-probability regions, while abnormal conditions happen in the low-probability regions. Thus, the prediction error of developed prediction models can be used to detect anomalies in the assessment indices.

A case is used for further explanation. A WT suffered a sudden breakdown on June 15, 2012 because of the severe oxidation of its carbon brush. An analysis of the error records in the SCADA system revealed that an error event of the generator bearing occurred on June 12, 2012.

Based on 3 $\sigma$-principle of normal distribution, the specific limits of prediction errors, which are set to $\pm 3 \sigma$, are used for detecting anomalies in the assessment indices. The prediction results of generator bearing $\boldsymbol{b}$ temperature and gearbox-side main bearing temperature are analyzed as an example. Figs. 6 and 7 show the prediction errors of the two assessment indices between May 13 and June 15, 2012. From May 13 to May 31, the prediction errors change in a small range centered at 0 . The first anomalies in the two indices are detected at an early stage on June 1. From June 1 to June 12, the prediction errors start increasing continuously, which are significantly greater than before. After the occurrence of the error event (i.e. June 12, 2012), the prediction errors rapidly increase and frequently exceed the value of $3 \sigma$

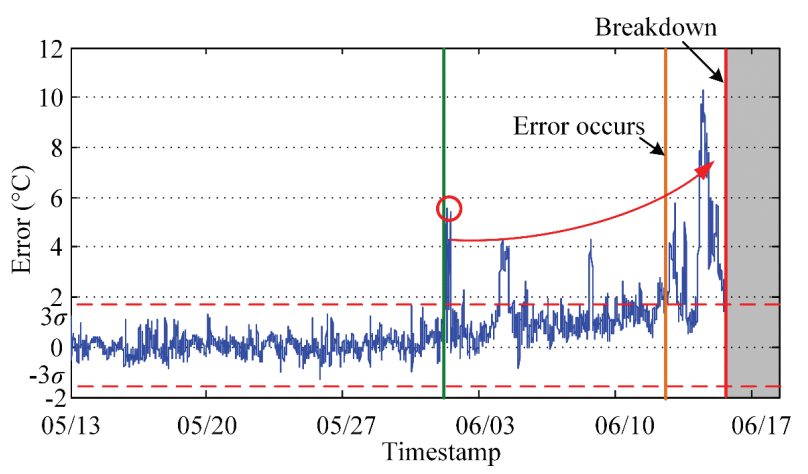

Fig. 6. Prediction errors of generator bearing $\boldsymbol{b}$ temperature

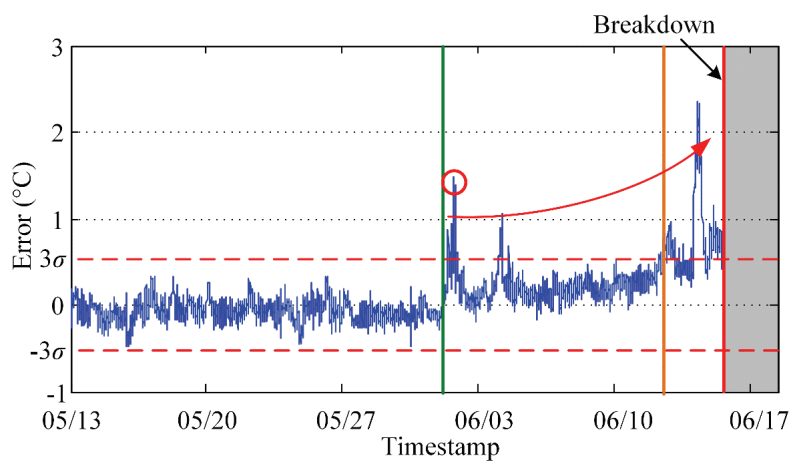

Fig. 7. Prediction errors of gearbox-side main bearing temperature. 


\section{Abnormal Level Quantification}

In this work, the ALI is defined to quantify the abnormal level of the proposed assessment indices. The ALI has a value in the range between 0 and 1, with a bigger value indicating a higher level of abnormal condition of the index.

\subsection{ALIs of the Type 1 and Type 2 indices}

The calculation of ALIs of the Type 1 and Type 2 indices is based on the principle that normal instances occur in high-probability regions, while abnormal conditions happen in the low-probability regions. The error residuals of prediction models for Type 1 and Type 2 indices are divided into four regions according to the probability density functions (PDFs) of zero-centered normal distribution and $3 \sigma$-rule, as shown in Fig. 8. A fixed-size moving window is applied to obtain the prediction error series. A window size of 144 points, which is 24-hour error residuals, is considered. The moving step of the time window is 6-hours. ALI is calculated as follows:

$$
g_{1}=1-\frac{R_{i} n_{i}}{\sum_{i=1}^{4} R_{i} n_{i}}
$$

where $n_{k}$ is the number of data in the $i$ th region and $R_{i}$ is the penalty factor, which is set to be $\{1,2,3,4\}$.

\subsection{ALIs of the type 3 indices}

Parzen estimation, a commonly used nonparametric estimation method, is used to describe the normal behaviors of Type 3 indices. Cumulative probability distribution functions (CPDFs) of the parameter values can be expressed as follows [41]:

$$
P(x)=\frac{1}{h n} \sum_{i=1}^{n} K\left(\frac{x-x_{i}}{h}\right)
$$

where $\left\{x_{1}, \ldots, x_{n}\right\}$ is a series of sample data, $K$ is the kernel function, and $h$ is the window width. The Gaussian kernel

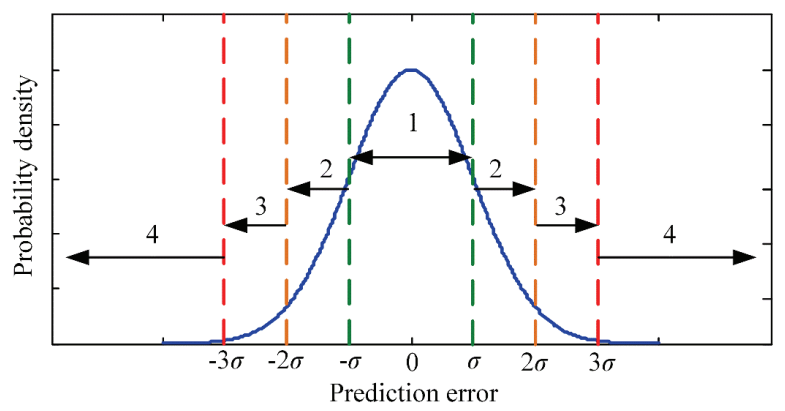

Fig. 8. Four regions of prediction errors of the NN based prediction models is used and the approximate mean integrated square error (AMISE) based approach is applied to obtain the optimal window width [42].

Because the Type 3 indices change significantly in a short time, data with an interval of $1 \mathrm{~min}$ are used. Data for three months are collected when the WT is healthy, which are used to obtain the Parzen estimation based CPDFs. The estimated CPDF for yaw angle error of a WT is taken as an example, as shown in Fig. 9.

The prediction errors are divided into three regions according to the quantile $0.025,0.25,0.75$ and 0.975 , as shown in Fig. 9. A fixed-size moving window is also used. The window size is set to 1440 points to obtain data in 24 hours and the moving step is 6-hours. ALIs of the Type 3 indices can be calculated as:

$$
g_{2}=1-\frac{R_{k} n_{k}}{\sum_{k=1}^{3} R_{k} n_{k}}
$$

where $n_{k}$ is the number of data in the $k$ th region and $R_{k}$ is the penalty factor, which is set to be $\{1,2,3\}$.

\section{The BPNN Model for WT Condition Assessment}

\subsection{WT condition division}

Currently, there are no generalized rules for division of WT conditions. In order to provide an early alarming prior to fault occurrences, the operating conditions of WTs are divided into three categories:

"Normal" condition: "Normal" condition indicates the WT can be considered healthy. During the period of "Normal" condition, the prediction errors of Type 1 and Type 2 indices change in a small range centered at 0 and very few of them exceed the specified limits (i.e. $\pm 3 \sigma$ ). The indices of Type 3 show small fluctuations under the "Normal" condition.

"Alert" condition: "Alert" condition denotes the status between the "Normal" condition and the "Dangerous" condition of WTs, which is an early indication of developing faults of WTs. During the period of "Alert"

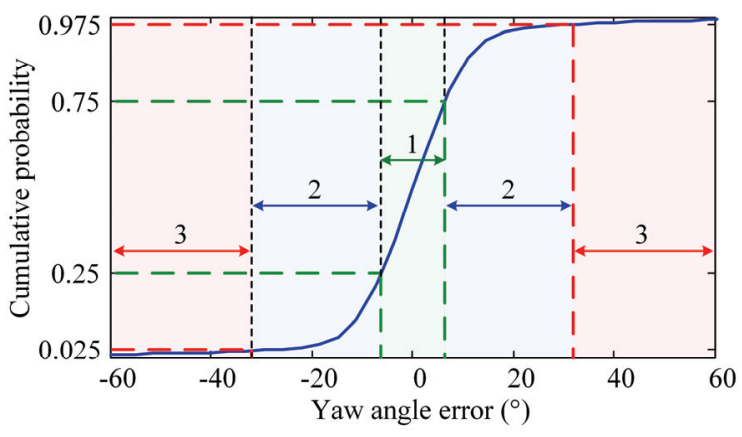

Fig. 9. Three regions of yaw angle error 
condition, prediction errors of some assessment indices show an obvious increasing tendency. Some of the prediction errors occasionally exceed the specified limits (i.e. $\pm 3 \sigma$ ). For some WT faults such as the yaw system fault and hydraulic system fault, some of the Type 3 indices show a tendency to reach their specified threshold values under the "Alert" condition.

"Dangerous" condition: "Dangerous" condition denotes the WT runs with the fault. During the period of "Dangerous" condition, the prediction errors of some indices rapidly increase and frequently exceed the specified limits (i.e. $\pm 3 \sigma$ ).

\subsection{The BPNN based assessment model}

NNs have been widely used in pattern recognition, nonlinear optimization, automatic target recognition, robotics, expert systems and many other fields. In this study, the BPNN algorithm is used for the condition assessment of WTs. Fig. 10 shows the structure diagram of NN model for condition assessment. The inputs to the BPNN are the ALIs of the assessment indices. Since there are 21 indices for monitoring WT conditions (Table 1), the input layer has 21 neuron nodes. The weight of the established BPNN model was optimized using the Levenberg-Marquardt iterative algorithm [43]. The transfer functions used in the hidden and the output layers are the tan-sigmoid and the linear functions, respectively. The output layer owns 3 neuron nodes corresponding to the three WT operating conditions (i.e. "Normal", "Alert", and

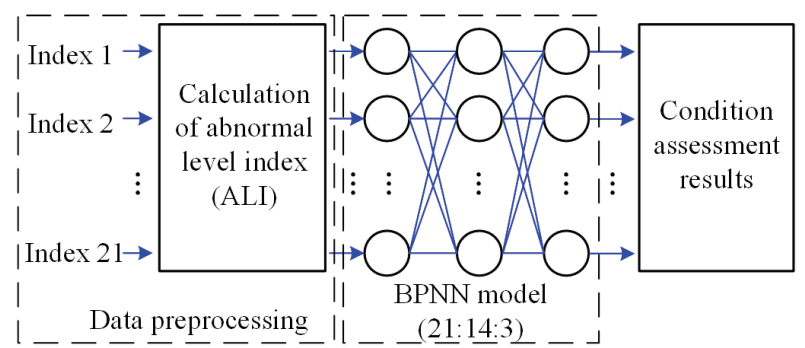

Fig. 10. Structure diagram of BPNN model for condition assessment

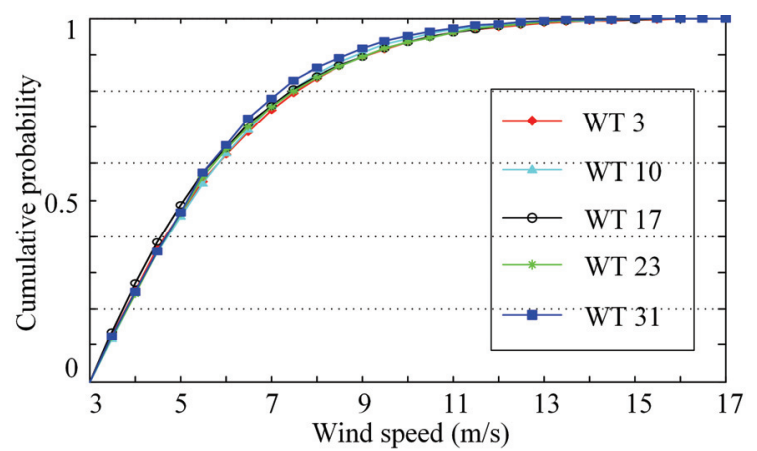

Fig. 11. Wind speed probability distributions of five WTs
"Dangerous"), which is denoted as $\boldsymbol{O}=\left(o_{1}, o_{2}, o_{3}\right)^{T}=(1,0$, $0)^{T},(0,1,0)^{T}$, and $(0,0,1)^{T}$, respectively. The condition assessment result of the WT corresponds to the maximal value of the $\boldsymbol{O}$ vector: $o_{\max }=\max \left(o_{j}\right)(j=1,2,3)$.

\subsection{Data sampling and network construction}

"Normal" condition: Fig. 11 shows the wind speed probability distributions at several WTs in the studied wind farm. It can be seen that most of the wind speed data are distributed in the range from $3 \mathrm{~m} / \mathrm{s}$ to $17 \mathrm{~m} / \mathrm{s}$. For the capability of the assessment model for generalization, the data selection for WTs classified under the "Normal" condition is conducted as follows: The range of the wind speed is divided into 14 intervals (i.e. $3 \mathrm{~m} / \mathrm{s}$ to $4 \mathrm{~m} / \mathrm{s}, 4 \mathrm{~m} / \mathrm{s}$ to $5 \mathrm{~m} / \mathrm{s}, \cdots, 16 \mathrm{~m} / \mathrm{s}$ to $17 \mathrm{~m} / \mathrm{s}$ ). By applying the fixed-size moving window (seen in Section 4), the ALIs of assessment indices and the 24-hour average wind speed can be calculated. Based on this, a datum from each wind speed interval is randomly selected. All selected data are collected to form a data group, with each group containing 14 data points. A total of 25 data groups are randomly selected, amounting to 350 data points.

"Alert" condition: The assessment index is considered abnormal if more than three subsequent values of the prediction error exceed the allowable limits (i.e. $\pm 3 \sigma$ ). ALIs of the assessment indices during the period from the first anomaly to the occurrence of the fault are selected as the "Alert" data. In this work, the same amount of data points are selected for each fault, yielding 350 data points.

"Dangerous" condition: "Dangerous" data indicate the condition of WTs running with fault. Based on ALIs of the assessment indices during "Dangerous" condition, the same number of data points are chosen for each fault, resulting in 350 data points.

Different three-layer BPNNs have been trained to determine an appropriate number of nodes in the hidden

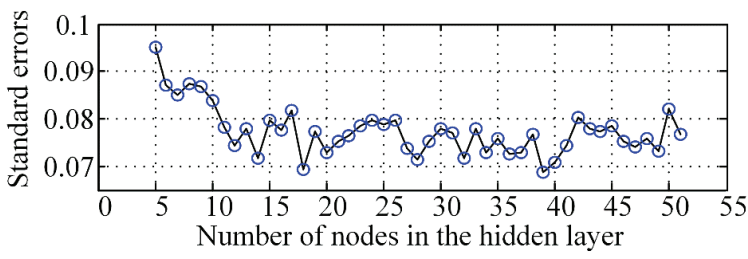

(a) Standard errors with different nodes in the hidden layer

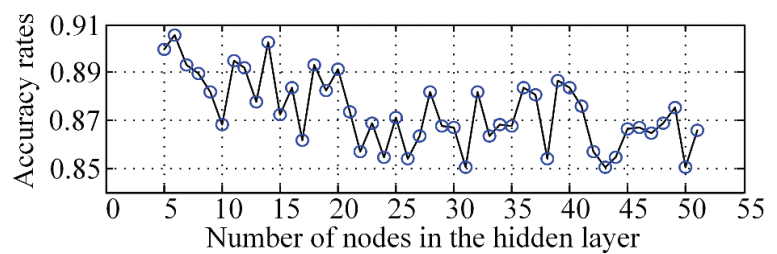

(b) Accuracy rates with different nodes in the hidden layer

Fig. 12. Results of BPNN with different nodes in the hidden layer 
layer. Given that there is no exact formula to determine the number of nodes in the hidden layer, neural networks with 5 to 51 nodes in the hidden layer are trained. Consequently, 47 types of BPNN models have been trained and compared, and every model is trained and tested for 20 times to find the best network structure among the 47 possible models. The sample data of each condition (350 data points) are randomly divided into 19 groups (266 data points) of training data and 6 groups ( 84 data points) of test data for each time. The standard errors and the accuracy rates are obtained in the tests. The average value of the two training parameters is then obtained, as seen in Fig. 12. The criterion of the accuracy rate is based on the following conditions: If $\left|o_{1}-d_{1}\right|<0.1$ and $\left|o_{2}-d_{2}\right|<0.1$ and $\mid o_{3}-d_{3}$ $\mid<0.1$, the output is correct; otherwise, the output is wrong. The standard error are calculated as:

$$
S=\sqrt{\frac{1}{3 n} \sum_{i=1}^{n}\left[\left(o_{1}^{i}-d_{1}^{i}\right)^{2}+\left(o_{2}^{i}-d_{2}^{i}\right)^{2}+\left(o_{3}^{i}-d_{3}^{i}\right)^{2}\right]}
$$

where $S$ is the standard error of the results of the assessment model; $\boldsymbol{O}^{i}=\left(o_{1}{ }^{i}, o_{2}{ }^{i}, o_{3}{ }^{i}\right)^{\mathrm{T}}$ is the vector of the $i$-th testing result; and $\boldsymbol{D}^{i}=\left(d_{1}{ }^{i}, d_{2}{ }^{i}, d_{3}{ }^{i}\right)^{\mathrm{T}}$ is the vector of expected output.

By considering the standard errors and the accuracy rates, the BPNN based assessment model with 14 nodes in the hidden layer exhibits the better performance, which is used for the condition assessment model in this paper.

\section{Verification and Analysis}

Two cases are selected to validate the proposed WT condition assessment method. In case 1, the impact of data preprocessing on the effectiveness of condition assessment is analyzed. The proposed BPNN model is compared with the radial basis function neural network (RBFNN) model, the least square support vector machine (LS-SVM) [44] model, and the $k$-NN model in case 2 .

\subsection{Case 1}

The accuracies of the NN Model trained by the standardized data (Model 1) and the model trained by the preprocessed data (Model 2) are compared. Fig. 13 shows the analysis flowchart. The standardized method is given as (9). The test dataset (240 data points) contains three conditions: "Normal", "Alert", and "Dangerous" in the ratio 1:1:1. For the "Alert" and "Dangerous" conditions, the same amount of data points are selected for each fault. These data are randomly divided into 10 groups for testing. Table 6 shows the accuracies of Model 1 and Model 2. As seen in the table, Model 2 is better than Model 1, which indicates that the proposed data processing method can increase the accuracy of assessment model.
Table 6. Accuracies of Models 1 and 2.

\begin{tabular}{c|c|c}
\hline Test data & Accuracy of Model 1(\%) & Accuracy of Model 2(\%) \\
\hline Group 1 & 77.21 & 88.13 \\
Group 2 & 72.63 & 89.84 \\
Group 3 & 78.32 & 90.01 \\
Group 4 & 83.5 & 89.18 \\
Group 5 & 81.46 & 87.26 \\
Group 6 & 73.16 & 91.86 \\
Group 7 & 71.94 & 93.61 \\
Group 8 & 80.59 & 89.14 \\
Group 9 & 84.05 & 91.79 \\
Group 10 & 74.09 & 92.52 \\
Mean value & 77.70 & 90.33 \\
\hline
\end{tabular}

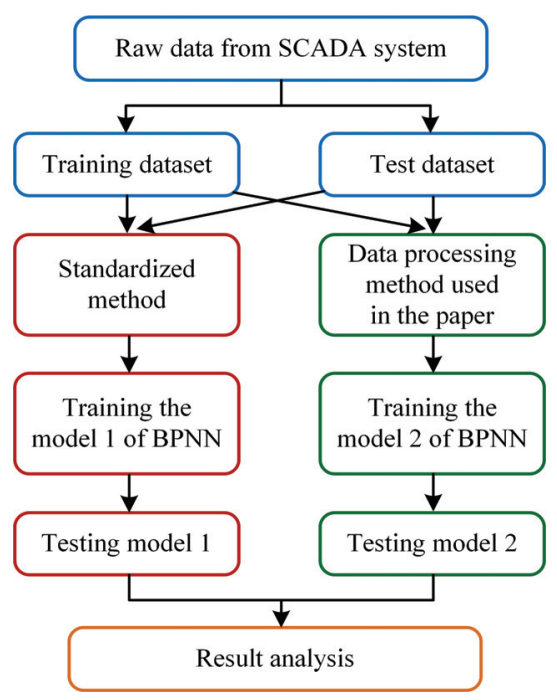

Fig. 13. Flowchart of the effect analysis of data processing

$$
Z_{i}=\frac{x_{i}-\bar{x}}{\sqrt{\frac{1}{n-1} \sum_{i=1}^{n}\left(x_{i}-\bar{x}\right)^{2}}}
$$

where $Z_{i}$ is the standardized data; $x_{i}$ is the measured value of the assessment index; and $\bar{x}$ is the mean value of the assessment index.

\subsection{Case 2}

The proposed BPNN model is compared with the RBFNN, the LS-SVM and the $k$-NN model is this case. To facilitate fair comparison, all the approaches are based on the same training samples. In RBFNN model, the spread of RBF plays an important role in successful application of neural networks. In this work, 10 -fold cross validation is applied to select the optimal spread among some candidate values, which ensures that the networks provide the best generalization performance. In this way, the spread value is chosen to be 5 in this study. For the LS-SVM model, the particle swarm optimization algorithm is used to select the optimal feature parameters by varying the values of penalty factor $\mathrm{C}$ and kernel function parameters $\sigma$ in the ranges $\left[\begin{array}{ll}1 & 10^{2}\end{array}\right]$ and $\left[\begin{array}{ll}10^{-1} & 10^{2}\end{array}\right][45]$. The best parameters obtained 
Table 7. Performance comparisons.

\begin{tabular}{c|c|c}
\hline Model & Average accuracy $(\%)$ & Average testing time $(\mathrm{s})$ \\
\hline BPNN & 90 & 0.0084 \\
RBFNN & 87.08 & 0.1532 \\
LS-SVM & 85.42 & 3.3598 \\
$k$-NN & 81.58 & 1.7805 \\
\hline
\end{tabular}
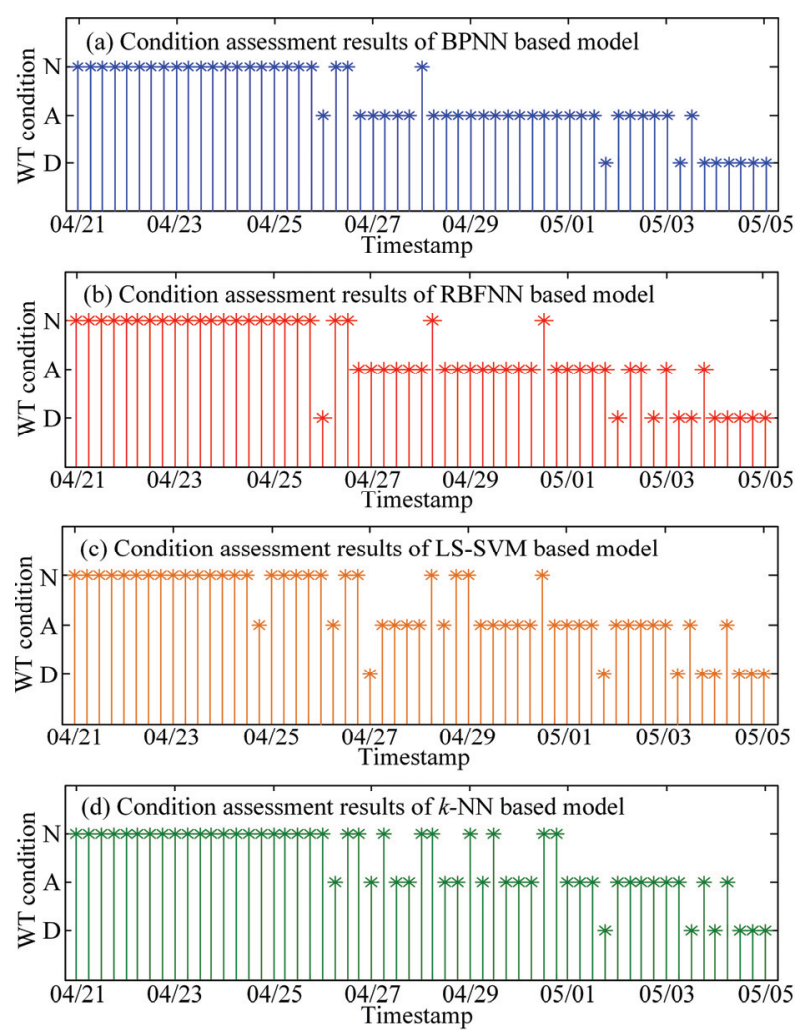

Fig. 14. Condition assessment results of a WT from April 21 to May 05

for the LS-SVM model are $\mathrm{C}=93.68, \sigma^{2}=5.31$. For the $k$ $\mathrm{NN}$ model, the Euclidean distance metric is selected and 10 -fold cross validation is employed to find the optimal $k$ value among some candidate values. The best level is achieved with the parameter $k=15$.

The four models have been tested 10 times by using the 10 groups of test data used in case 1 . The average accuracy and average time consumptions are shown in Table 7. It can be seen that the BPNN model has the best accuracy with the shortest computing time.

The condition assessment results regarding the four assessment models for a fault of WT generator on May 3, 2012 and a fault of WT gearbox on July 23, 2012, are taken as an example, as shown in Figs. 14 and 15. It can be seen that the BPNN model can exactly identify the "Alert" and "Dangerous" condition of WT before the generator is out of service. However, many vibrations have been found in the results of the RBFNN, LS-SVM and $k$-NN model, meaning that those three models may delay to identify the "Alert" and "Dangerous" conditions. The BPNN model is more stable and with less misjudgment of WT operating
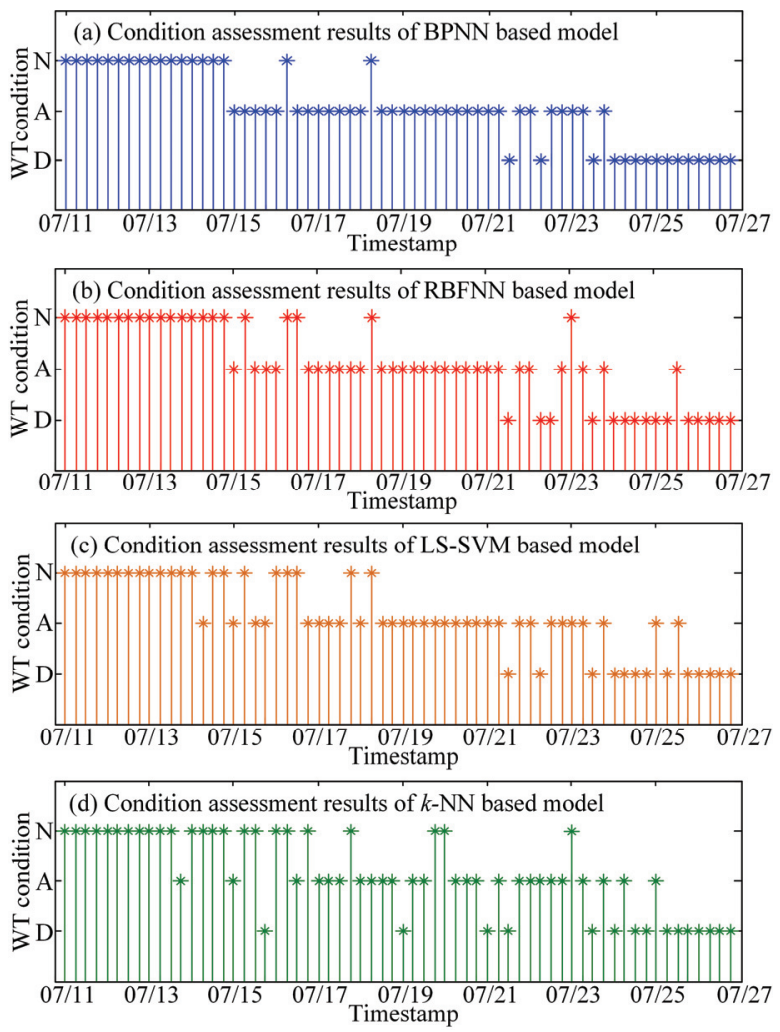

Fig. 15. Condition assessment results of a WT from July 11 to July 27

conditions.

As shown in Figs. 14 and 15, the 'Alert' condition can give an early warning of dangerous WT faults. The durations of the 'Alert' conditions vary and are dependent on the fault types. The 'Alert' condition in the fault of generator lasted for about 6 days in Fig. 14 while the 'Alert' condition lasted about 8 days before the actual gearbox fault happened, shown in Fig. 15.

\section{Conclusions}

This paper presented a novel approach for WT condition assessment by using SCADA data. Based on the monitoring data of a wind farm SCADA system, the indices for WT condition assessment were determined and classified into three categories. According to the characteristics of the different indices, the NN based prediction models were developed to extract the useful information from the SCADA data to further increase the accuracy of WT condition assessment. A new index called ALI is proposed to quantify the abnormal level of WT assessment indices. The ALIs were used as the inputs to the BPNN based assessment model.

The BPNN based condition assessment method was validated using the online data of real $1.5 \mathrm{MW}$ WTs. The results show that the proposed data processing method can increase the accuracy of assessment model. The BPNN 
model could effectively estimate the operating conditions of WTs and has been verified to be more accurate than the RBFNN model, the LS-SVM model and the $k$-NN model in WT condition assessment. The "Alert" condition of WTs discussed in this paper for early fault alarming has demonstrated to be useful in developing proper maintenance strategies for wind farms. In future research we will focus on enhancing the applicability of the proposed assessment method in large wind farms. Furthermore, other operating and fault conditions will be investigated. The warning time with respect to different WT faults also deserves further study.

\section{Acknowledgements}

This work was supported in part by National Key Basic Research Program of China (973 Program) (2012CB215205), the Specialized Research Fund for the Doctoral Program of Higher Education of China (SRFDP) (20110191130004), the visiting scholar fund of State Key Lab of Power Transmission Equipment and System Security (SKLPES), Chongqing University, China, the National Natural Science Foundation of China (no. 51321063), and the 111 Project of the Ministry of Education, China (B08036). The work of C. Wang was partially supported by the National Science Foundation of USA under Grants ECCS-1202133 and 1508910. Thanks to Mr. Michael Fornoff for proofreading the paper.

\section{References}

[1] Chehouri A and Younes R: "Review of performance optimization techniques applied to wind turbines", Applied Energy, vol. 142, pp. 361-388, 2015.

[2] Yuan XM: "Overview of problems in large-scale wind integrations", Journal of Modern Power Systems and Clean Energy, vol. 1, no. 1, pp. 22-25, 2013.

[3] Gil MDP, Gomis-bellmunt O, and Sumper A: "Technical and economic assessment of offshore wind power plants based on variable frequency operation of clusters with a single power converter", Applied Energy, vol. 125, no. 21, pp. 218-229, 2014.

[4] Yang WX, Tavner PJ, Crabtree CJ, Feng Y, and Qiu Y: "Wind turbine condition monitoring: technical and commercial challenges", Wind Energy, vol. 17, no. 5, pp. 673-693, 2014.

[5] Johan R and Margareta BL: "Survey of failures in wind power systems with focus on Swedish wind power plants during 1997-2005", IEEE Transactions on Energy Conversion, vol. 22, no. 1, pp. 167-173, 2007.
[6] Milborrow D: "Operation and maintenance costs compared and revealed", Wind Stats, vol. 19, no. 3, pp. 3, 2006.

[7] Caselitz P, Bussel GW, and Spinato F: "Rotor condition monitoring for improved operational safety of offshore wind energy converters", Journal of Solar Energy Engineering, vol. 127, no. 2, pp. 253-261, 2005.

[8] Soua S, Lieshout PV, Perera A, Gan TH, and Bridge B: "Determination of the combined vibrational and acoustic emission signature of a wind turbine gearbox and generator shaft in service as a prerequisite for effective condition monitoring", Renew Energy, vol. 51, no. 2, pp. 175-181, 2013.

[9] Becker E and Posta P: "Keeping the blades tunning: Condition monitoring of wind turbine gearbox", Refocus, vol. 7, no. 2, pp. 26-32, 2013.

[10] Verbruggen TW: "Wind turbine operation and maintence based on condition monitoring", WT- $\Omega$. Final Report, ECN-C-03-047, Energy Research Center, Netherlands, 2003.

[11] Schlechtingen M, Santos IF, and Achiche S: "Wind turbine condition monitoring based on SCADA data using normal behavior models. Part 1: System description", Applied Soft Computing Journal, vol. 13, no. 1, pp. 259-270, 2013.

[12] Kusiak A and Verma A: "A data-mining approach to monitoring wind turbines", IEEE Transactions on Sustainable Energy, vol. 3, no. 1, pp. 150-157, 2012.

[13] Nilsson J, Bertling L: "Maintenance management of wind power systems using condition monitoring systems - Life cycle cost analysis for two case studies", IEEE Transactions on Energy Conversion, vol. 22, no. 1, pp. 223-229, 2007.

[14] Kusiak A, Verma A and Wei XP: "Wind turbine capacity frontier from SCADA", Wind Systems Magazine, vol. 3, no. 9, pp. 36-39, 2012.

[15] Hameed Z, Hong YS, Cho YM, Ahn SH, and Song CK. "Condition monitoring and fault detection of wind turbines and related algorithms: A review", Wind Energy, vol. 13, no. 1, pp. 1-39, 2009.

[16] Lapira E, Brisset D, Ardakani HD, Siegel D, and Lee $\mathrm{J}$ : "Wind turbine performance assessment using multi-regime modeling approach", Renew Energy, vol. 45, no. 3, pp. 86-95, 2012.

[17] Schlechtingen M, Santos IF, and Achiche S: "Using data-mining approaches for wind turbine power curve monitoring: A comparative study", IEEE Transactions on Sustainable Energy, vol. 4, no. 3, pp. 671-679, 2013.

[18] Kusiak A, Zheng HY, and Song Z: "Models for monitoring wind farm power", Renewable Energy, vol. 34, no. 3, pp. 583-590, 2009.

[19] Marvuglia A, and Messineo A: "Monitoring of wind farms power curves using machine learning techniques", Applied Energy, vol. 98, pp. 574-583, 
2012

[20] Jia XD, Jin C, Buzza M, Wang W, Lee J: "Wind turbine performance degradation assessment based on a novel similarity metric for machine performance curves", Renewable Energy, vol. 99, pp. 1191-1201, 2016.

[21] Kusiak A and Verma A: "The prediction and diagnosis of wind turbine faults", Renewable Energy, vol. 36, no. 1, pp. 16-23, 2011.

[22] Lydia M, Kumar SS, Selvakumar AI, and Kumer GEP: "A comprehensive review on wind turbine power curve modeling techniques", Renewable and Sustainable Energy Reviews, vol. 30, no. 2, pp. 452460, 2014.

[23] Yang WX, Court R, and Jiang JS: "Wind turbine condition monitoring by the approach of SCADA data analysis", Renewable Energy, vol. 53, no. 9, pp. 365-376, 2013.

[24] Ata R: "Artificial neural networks applications in wind energy systems: a review", Renewable and Sustainable Energy Reviews, vol. 49, no. 534-562, 2015.

[25] Schlechtingen $M$ and Santos IF: "Comparative analysis of neural network and regression based condition monitoring approaches for wind turbine fault detection", Mechanical Systems \& Signal Processing, vol. 25, no. 5, pp. 1849-1875, 2011.

[26] Zaher A, McArthur SDJ, and Infield DG: "Online wind turbine fault detection through automated SCADA data analysis", Wind Energy, vol. 12, no. 6, pp. 574-593, 2009.

[27] Liu YQ, Shi J, Yang YP, Lee WJ: "Short-term windpower prediction based on wavelet transform-support vector machine and statistic-characteristics analysis", IEEE Transactions on Industry Applications, vol. 48, no. 4, pp. 1136-1141, 2012.

[28] Chen B, Peter CM, and Peter JT: "Automated on-line fault prognosis for wind turbine pitch systems using supervisory control and data acquisition", IET Renewable Power Generation, vol. 9, 503-513, 2015.

[29] Wang L, Zhang ZJ, Long H, Xu J, Liu RH: "Wind turbine gearbox failure identification with deep neural networks", IEEE Transactions on Industrial Informatics, to be published.

[30] Guo P, Infield D, and Yang XY: "Wind turbine generator condition-monitoring using temperature trend analysis", IEEE Transactions on Sustainable Energy, vol. 3, no. 1, pp. 124-133, 2012.

[31] Kusiak A and Verma A: "Monitoring wind farms with performance curves", IEEE Transactions on Sustainable Energy, vol. 4, no. 1, pp. 192-199, 2013.

[32] Cross P and Ma XD: "Nonlinear system identification for model-based condition monitoring of wind turbines", Renewable Energy, vol. 71, no. 11, pp. 166-175, 2014.

[33] Kusiak A, and Verma A: "Analyzing bearing faults in wind turbines: A data-mining approach", Renewable Energy, vol. 48, no. 6, pp. 110-116, 2012.

[34] Schlechtingen M, and Santos IF: "Comparative analysis of neural network and regression based condition monitoring approaches for wind turbine fault detection", Mechanical Systems \& Signal Processing, vol. 25, no. 5, pp. 1849-1875, 2011.

[35] Liao RJ, Zheng HB, and Grzybowski S: "An integrated decision-making model for condition assessment of power transformers using fuzzy approach and evidential reasoning", IEEE Transactions on Power Delivery, vol. 26, no. 2, pp. 11111118, 2011.

[36] Qian Z and Yan Y: "Fuzzy synthetic method for life assessment of power transformer", IEE Proceedings Science Measurement and Technology, vol. 151, no. 3, pp. 175-180, 2004.

[37] $\mathrm{Li} \mathrm{H}, \mathrm{Hu} \mathrm{YG}$, Chen Z, Ji HT, and Zhan B: "An improved fuzzy synthetic condition assessment of a wind turbine generator system", International Journal of Electrical Power and Energy Systems, vol. 45, no. 1, pp. 468-476, 2013.

[38] Yampikulsakul N, Byon E, Huang S, Sheng S, and You M: "Condition monitoring of wind power system with nonparametric regression analysis", IEEE Transactions on Energy Conversion, vol. 29, no. 2, pp. 288-299, 2014.

[39] Xiang DW, Ran L, Tavner $P$ and Bryant A: "Monitoring Solder Fatigue in a Power Module Using Case-Above-Ambient Temperature Rise", IEEE Transactions on Industry Applications, vol. 47, no. 6, pp. 2578-2591, 2012.

[40] Spera DA: "Wind turbine technology: fundamental concepts of wind turbine engineering", New York, ASME, USA (1994)

[41] Parzen E: "On the estimation of a probability density function and the mode", The Annals of Mathematical Statistics, vol. 33, no. 3, pp. 1065-1076, 1962.

[42] Silverman BW: "Density Estimation for Statistics and Data Analysis", New York, Chapman and Hall, USA, 1986.

[43] Parzen E: "On the estimation of a probability density function and the mode", The Annals of Mathematical Statistics, vol. 33, no. 3, pp. 1065-1076, 1962.

[44] Yin Z and Zhang JH: "Operator functional state classification using least-square support vector machine based recursive feature elimination technique", Computer Methods and Programs in Biomedicine, vol. 113, no. 1, pp. 101-115, 2014.

[45] Zheng HB, Liao RJ, Grzybowski S, and Yang LJ: "Fault diagnosis of power transformers using multiclass least square support vector machines classifiers with particle swarm optimization" IET Electric Power Applications, vol. 5, no. 9, pp. 691696, 2011. 


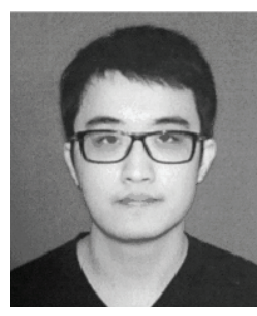

Peng Sun He received the Bachelor degree in electrical engineering in 2011, from Chongqing University, Chongqing, China. He is currently a Ph.D. degree candidate at Chongqing University. His major research interest is reliability assessment of electrical collector systems of wind farm.

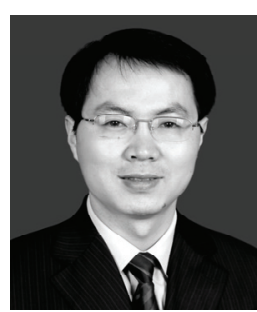

Jian Li He received his M.S. and Ph.D. degrees in electrical engineering in 1997 and 2001, respectively from Chongqing University, Chongqing, China. He is currently a professor and the head of High Voltage and Insulation Technology Department at Chongqing University. His major research interests include online detection of insulation condition in electrical devices, partial discharges, and insulation fault diagnosis for high voltage equipment, environment-friendly insulating liquid, anti-icing coating of insulator and transmission line, and polyethylene/ organic-montmorillonite nano-composites. He is an author and coauthor of more than 60 journal papers and 50 papers published in the proceedings of international conferences.

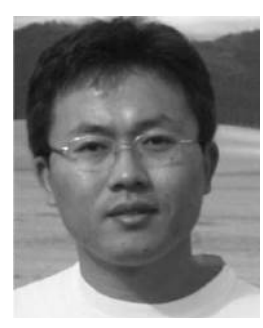

Caisheng Wang He received the B.S. and M.S. degrees from Chongqing University, Chongqing, China, in 1994 and 1997, respectively, and the Ph.D. degree from Montana State University, Bozeman, MT, USA, in 2006, all in electrical engineering. From 1997 to 2002, he was an Electrical Engineer and then a Vice Department Chair with the Zhejiang Electric Power Test and Research Institute, Hangzhou, China. Since 2006, he has been with Wayne State University, Detroit, MI, USA, where he is currently an Associate Professor with the Department of Electrical and Computer Engineering. His current research interests include modeling and control of power systems and electric vehicles, energy storage devices, distributed generation and microgrids, alternative/ hybrid energy power generation systems, and fault diagnosis and on-line monitoring of electric apparatus.

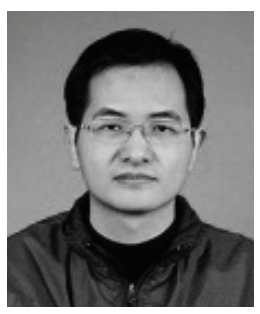

Yonglong Yan He received the M.S. degree in 2008, from Chongqing University, Chongqing, China. He is currently a Ph.D. student in school of Electrical Engineering at Chongqing University, China. His major research interests include fault diagnosis and condition assessment for wind power equipment, and reliability and optimization of maintenance strategy for wind farm. 